\title{
DENSITIES OF VALENCE ELECTRONS IN SIMPLE METALS EFFECTIVE WITH RESPECT TO ELECTRON-POSITRON CORRELATIONS
}

\author{
G. Kontrym-Sznajd and A. Rubaszek \\ W. Trzebiatowski Institute of Low Temperature and Structure Research \\ Polish Academy of Sciences \\ P.O. Box 937, 50-959 Wrocław, Poland
}

The influence of the electron-positron interactions on the momentum density of annihilation quanta in real metals is discussed. The role of momentum dependence of two-particle electron-positron correlations is set forth. The effective densities describing electron-positron correlations are presented for valence electrons in simple metals.

PACS numbers: $78.70 . B j, 71.20 .-b$

\section{Introduction}

In studies of momentum densities of electron-positron $2 \gamma$ annihilation quanta $\rho(p)$ in real metals, the local density approach (LDA) has been recently quite often applied (for reviews see, e.g., [1]). The results for annihilation characteristics obtained within LDA, however, are as reliable as far the assumed electron-positron correlation functions following from the electron gas theory are correct. Experimental positron annihilation data in simple metals would allow for verification of annihilation parameters in jellium obtained within various theories (reviewed in [1]).

The problem is how to state the density of electron gas in real metal in order to compare experimental and theoretical correlation functions, and how far this comparison is possible. In this work we show that for valence electrons in simple metals the effective densities of an electron gas are well defined and may be determined in an unique way. As the result, the whole shape of $\rho(p)$ inside the central Fermi surface (FS) may be determined, basing on the independent particles model (IPM) results and electron-positron enhancement factors in an electron gas, $\varepsilon_{\text {jell }}\left(p, r_{\mathbf{s}}^{\text {eff }}\right)$, if only the effective density parameter $r_{\mathbf{s}}^{\text {eff }}$ is known. 


\section{Theory and results}

If the rare-gas core contribution to annihilation is neglected, the momentum density of $2 \gamma$ annihilation quanta is given by the formula

$$
\rho(\boldsymbol{p})=\frac{\pi r_{0}^{2} c}{\Omega} \sum_{\boldsymbol{k}_{j}} n(\boldsymbol{k} j)\left|\int_{\Omega} \mathrm{e}^{-\mathrm{i} \boldsymbol{p} \cdot \boldsymbol{r}} \psi_{\boldsymbol{k} j}^{\mathrm{ep}}(\boldsymbol{r}, \boldsymbol{r}) \mathrm{d} \boldsymbol{r}\right|^{2},
$$

where $\Omega, r_{0}$, and $c$ are the volume of the unit cell, classical electron radius, and velocity of light, respectively, $n(k j)$ denotes the occupation number of the electronic state $k j$ in the host material, and $\psi_{k j}^{\mathrm{ep}}\left(r_{\mathrm{e}}, r_{\mathrm{p}}\right)$ is the electron-positron pair wave function of an electron in the initial state $k j$ located at $r_{\mathrm{e}}$ and the thermalised positron at $r_{\mathrm{p}}$. Momenta $\boldsymbol{p}$ are considered in the extended zone scheme, while $\boldsymbol{k}$ are in the first Brillouin zone (1BZ).

The total annihilation rate $\lambda(\lambda=1 / \tau$, where $\tau$ is the positron lifetime) is the normalization constant of Eq. (1), i.e.

$$
\lambda=\int \mathrm{d} p \rho(p)=\pi r_{0}^{2} c \int \mathrm{d} r\left(\sum_{k_{j}} n(k j)\left|\psi_{k j}^{\mathrm{ep}}(r, r)\right|^{2}\right) .
$$

For $r_{\mathrm{e}}=r_{\mathrm{p}}$ the two-particle function $\psi_{k_{j}}^{\mathrm{ep}}\left(r_{\mathrm{e}}, r_{\mathrm{p}}\right)$ may be written in the form [2-4] (this notation has been also repeated in Ref. [5])

$$
\psi_{k j}^{\mathrm{ep}}(r, r)=\psi_{k j}^{\mathrm{e}}(r) \psi_{+}(r) f(r)
$$

where

$$
\psi_{k j}^{\mathrm{e}}(r)=\frac{1}{\sqrt{\Omega}} \mathrm{e}^{\mathrm{i} \boldsymbol{k} \cdot \boldsymbol{r}}\left[\sum_{\boldsymbol{G}} u_{k_{j}}(\boldsymbol{G}) \mathrm{e}^{\mathrm{i} G \cdot \boldsymbol{r}}\right]
$$

is the electron wave function in the host material,

$$
\psi_{+}(r)=\frac{1}{\sqrt{\Omega}} \sum_{\boldsymbol{G}} \psi_{+}(\boldsymbol{G}) \mathrm{e}^{\mathrm{i} \boldsymbol{G} \cdot \boldsymbol{r}}
$$

stands for a positron wave function, and

$$
f_{k_{j}}(r)=\sum_{\boldsymbol{G}} f_{k j}(\boldsymbol{G}) \mathrm{e}^{\mathrm{i} G \cdot \boldsymbol{r}}
$$

are periodic in the lattice (cf. Ref. [4]) two-particle electron-positron correlation functions. Vectors $G$ in Eqs. (4) are the reciprocal-lattice vectors.

Within IPM $f_{k j}^{\mathrm{IPM}}(r) \equiv 1$ and IPM momentum density reads as

$$
\begin{gathered}
\rho^{\mathrm{IPM}}\left(p=k+G^{*}\right)=\sum_{k j} n(k j)\left|\phi_{k j}^{\mathrm{IPM}}\left(G^{*}\right)\right|^{2} \\
=\left|\phi_{k_{j} *}^{\mathrm{IPM}}\left(G^{*}\right)\right|^{2}\left[n\left(k j^{*}\right)+\alpha\left(k j^{*}, G^{*}\right)\right]
\end{gathered}
$$

(the term $\pi r_{0}^{2} c / \Omega$ has been omitted for convenience), where $\phi_{k_{j}}^{\mathrm{IPM}}(G)$ are the convolutions of $u_{k_{j}}(\boldsymbol{G})$ and $\psi_{+}(\boldsymbol{G})$, i.e.

$$
\phi_{k j}^{\mathrm{PPM}}(\boldsymbol{G})=\sum_{\boldsymbol{H}} u_{k j}(\boldsymbol{H}) \psi_{+}(\boldsymbol{G}-\boldsymbol{H}),
$$


and

$$
\alpha\left(k j^{*}, G^{*}\right)=\sum_{j \neq j^{\star}} n(k j)\left|\frac{\phi_{k_{j}}^{\mathrm{IPM}}\left(G^{*}\right)}{\phi_{l_{k^{*}} \cdot}^{\mathrm{IPM}}\left(G^{*}\right)}\right|^{2}
$$

are the corrections describing the band effects [4]. For nearly-free electron (NFE) populations (e.g., valence electrons in simple metals), for any fixed state $k j$ (except for those close to the $\mathrm{BZ}$ boundary) there is only one vector $G(j)$ such that (for more details see Ref. [4])

$$
\left|u_{k j}[G(j)]\right| \gg\left|u_{k_{j}}(G)\right| \text { for } \boldsymbol{G} \neq \boldsymbol{G}(j),
$$

while

and

$$
\left|u_{k^{*}}\left(G^{*}\right)\right| \gg\left|u_{k_{j}}\left(G^{*}\right)\right| \text { for } j \neq j^{*}
$$

$$
G\left(j^{*}\right)=G^{*}
$$

Using the methods of Ref. [4] it is easy to show that Eqs. (7) provide $\left|\alpha\left(k j^{*}, G^{*}\right)\right| \ll 1$. Thus, for momenta $p$ inside the FS (i.e. for $\left.n\left(k j^{*}\right)=1\right), \rho^{\text {IPM }}$ may be approximated within NFE as

$$
\rho_{\mathrm{NFE}}^{\mathrm{IPM}}\left(p=k+G^{*}\right) \approx\left|\phi_{k_{j} *}^{\mathrm{IPM}}\left(G^{*}\right)\right|^{2} .
$$

In the interacting system we have

$$
\begin{aligned}
\rho(p & \left.=k+G^{*}\right)=\sum_{k_{j}} n(k j)\left|\sum_{G} \phi_{k_{j}}^{\mathrm{IPM}}(G) f_{k_{j}}\left(G^{*}-G\right)\right|^{2} \\
& =\left|\phi_{k_{j} j^{*}}^{\mathrm{IPM}}\left(G^{*}\right)\right|^{2}\left|f_{k_{j} *}(0)\right|^{2}\left[1+\beta\left(k j^{*}, G^{*}\right)\right]\left[n\left(k j^{*}\right)+\xi\left(k j^{*}, G^{*}\right)\right]
\end{aligned}
$$

where

$$
\beta\left(k j^{*}, G^{*}\right)=\left|\sum_{G \neq G^{*}} \frac{\phi_{k_{j^{*}}}^{\mathrm{IPM}}(G)}{\phi_{k_{j^{*}}}^{\mathrm{IPM}}\left(G^{*}\right)} \frac{f_{k_{j^{*}}}\left(G^{*}-G\right)}{f_{k_{j^{*}}}(0)}\right|^{2}
$$

and $\xi\left(k j^{*}, G^{*}\right)$ are also small corrections for strongly delocalised electrons, if only $\left|f_{k_{j}}(0)\right| \gg\left|f_{k_{j}}(G \neq 0)\right|$, as it takes place e.g. within LDA (cf. Ref. [4]). As the result, for momenta $p$ inside the FS

$$
\rho_{\mathrm{NFE}}\left(p=k+G^{*}\right) \approx\left|\phi_{k_{j} *}^{\mathrm{IPM}}\left(G^{*}\right)\right|^{2}\left|f_{k_{j}}(0)\right|^{2}
$$

and

$$
\varepsilon_{\mathrm{NFE}}\left(p=k+G^{*}\right)=\rho_{\mathrm{NFE}}(p) / \rho_{\mathrm{NFE}}^{\mathrm{IPM}}(p) \approx\left|f_{k^{*}}(0)\right|^{2} .
$$

From formula (10b) it clearly follows that the momentum dependence of the electron-positron enhancement factors $\varepsilon(p)$ inside the FS is due to the state selectivity of two-particle correlation functions $f_{k_{j}}(r)$ only. Neither the form of the positron wave function nor state independent correlations $f(r)$ can change visibly the shape of $\rho(p)$ inside the FS with respect to $\rho^{\mathrm{IPM}}(p)$ for delocalised electronic populations (e.g. valence electrons in simple metals). 
Within LDA Eq. (10b) reads as

$$
\begin{aligned}
& \varepsilon_{\mathrm{NFE}}\left(p=k+G^{*}\right) \approx \frac{1}{\sqrt{\Omega}}\left|\int_{\Omega}\left\{\varepsilon_{\text {jell }}\left[E_{k_{j} \cdot} / E_{\mathrm{F}}, r_{\mathrm{s}}(r)\right]\right\}^{12} \mathrm{~d} r\right|^{2} \\
& =\varepsilon_{\text {jell }}\left[E_{k_{j^{*}}} / E_{\mathrm{F}}, r_{\mathrm{s}}\left(r^{*}\right)\right] .
\end{aligned}
$$

In Fig. 1 the effective electron densities $r_{s}\left(r_{0}\right), r_{s}\left(r_{1}\right)$, and $r_{s}\left(r_{\lambda}\right)$ are presented, where $r_{0}$ and $r_{1}$ are obtained according to Eq. (11) for momenta $|p|=0$ and

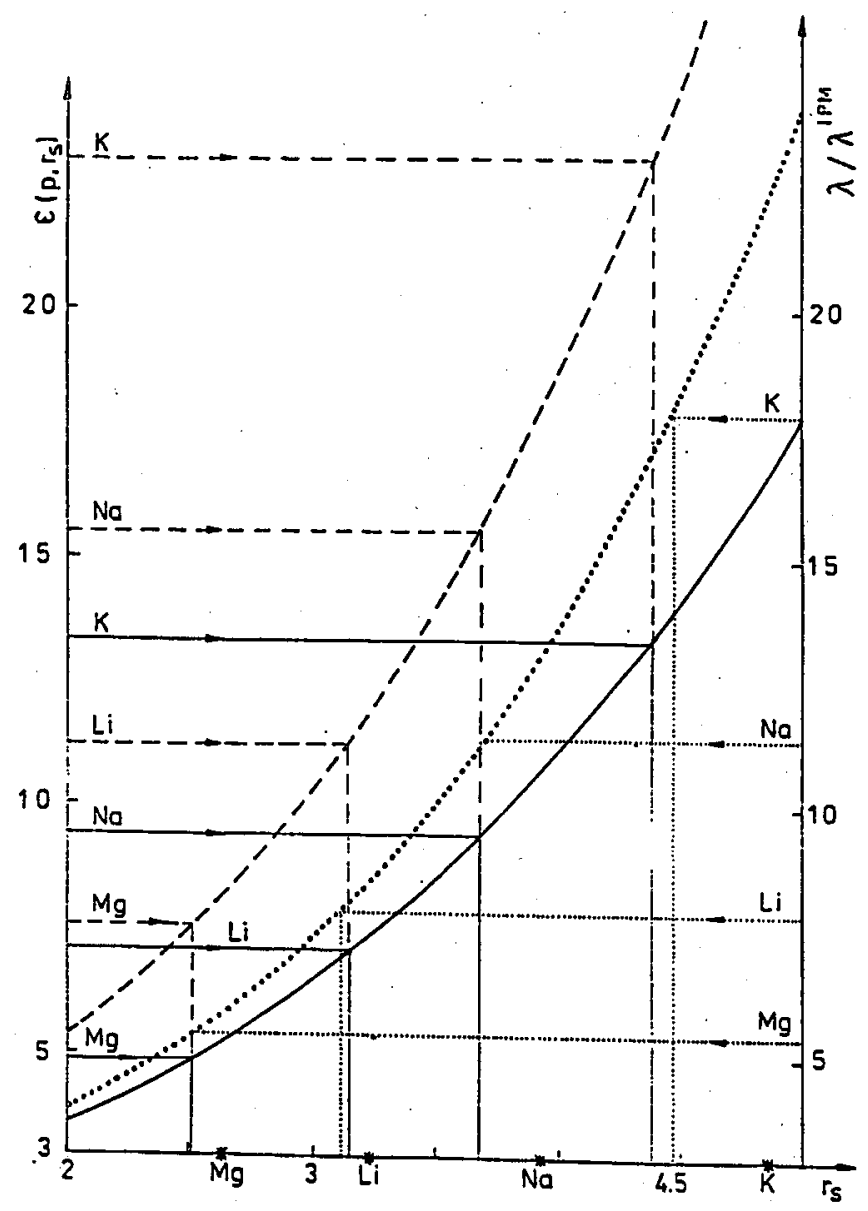

Fig. 1. Effective electron density parameters in $\mathrm{Mg}, \mathrm{Li}, \mathrm{Na}$, and $\mathrm{K}$ following from comparison of (a) $\varepsilon(0)$ with $\varepsilon_{\text {jell }}\left(0, r_{\mathrm{s}}\right)$ (solid lines), (b) $\varepsilon\left(p_{\mathrm{F}}\right)$ with $\varepsilon_{\text {jell }}\left(p_{\mathrm{F}}, r_{\mathrm{s}}\right)$ (dashed lines), (c) $\lambda_{\text {val }} / \lambda_{\text {val }}^{\mathrm{IPM}}$ with $g\left(r_{s}\right)=\lambda_{\text {jell }}\left(r_{s}\right) / \lambda_{\text {jell }}^{\mathrm{IPM}}\left(r_{\mathrm{s}}\right)$ (dotted lines). Values of $\varepsilon(p)$ and $\lambda_{\text {val }}$ in real metals were obtained within LDA (Refs. [6] and [3], respectively) while $\varepsilon_{\text {jell }}$ and $g\left(r_{s}\right)$ are results for an electron gas (Refs. [7] and [8], respectively). The a verage densities of valence electrons, $r_{s}^{\text {free }}$, are marked by stars. 
$\left|p / p_{\mathrm{F}}\right|=1$, respectively. The values of $\varepsilon(p)$ are based on the band structure calculations performed within linear muffin tin orbitals (LMTO) a verage sphercs approximation (ASA) (cf. Ref. [6]), and $\varepsilon_{\text {jell }}\left(p, r_{\mathrm{s}}\right)$ is the jellium result of Ref. [7]. Density parameter $r_{\mathrm{s}}\left(r_{\lambda}\right)$ was obtained in the same way, by comparing the correlation factor for total valence annihilation rates in real metals, $\gamma^{\text {corr }}=\lambda_{\text {val }} / \lambda_{\text {val }}^{\mathrm{IPM}}$, extracted from Ref. [3] with its jellium analog $\gamma_{\text {jell }}^{\text {corr }}\left(r_{s}\right)$, parametrised in Ref. [8]. It is visible that the values of $r_{\mathbf{s}}\left(r_{0}\right), r_{\mathbf{s}}\left(\boldsymbol{r}_{1}\right)$, and $r_{\mathbf{s}}\left(r_{\lambda}\right)$ are very close to each other, determining the effective electron density parameters $r_{\mathrm{s}}^{\text {eff }}$ describing electron-positron correlations for valence electrons in simple metals. These effective densities differ from the average valence electron densities in the Wigner-Seitz cell, described by $r_{\mathrm{s}}^{\text {free }}$ (marked by stars in Fig. 1). This agreement of $r_{\mathrm{s}}\left(\boldsymbol{r}_{0}\right), r_{\mathrm{s}}\left(r_{1}\right)$ and $r_{\mathrm{s}}\left(\boldsymbol{r}_{\lambda}\right)$ is the great advantage in the studies of electronic structure of materials by positron annihilation method; knowing the total annihilation rates in real metals, $\lambda_{\text {val }}$ and $\lambda_{\text {val }}^{\mathrm{IPM}}$, we are able to determine $r_{\mathrm{s}}\left(\boldsymbol{r}_{\lambda}\right) \approx r_{\mathrm{s}}^{\text {eff }}$ and read $\rho(p)$ for $p \in$ FS directly from $\rho^{\mathrm{IPM}}(p)$ as $\rho(p) \approx \rho^{\mathrm{IPM}}(p) \varepsilon_{\text {jell }}\left(p, r_{\mathrm{s}}^{\mathrm{eff}}\right)$, avoiding laborious calculations of convolutions of electron and positron wave functions with correlation functions.

It should be stressed here that for more localised electronic populations (e.g. $d$-or rare-gas core electrons) the above procedure is not applicable. In non-simple metals the effective electron densities may be defined in several various alternative ways (each of them substantiated in similar degree), depending on the effects under study. For this reason the great deal of caution is necessary when one tries to determine the effective electron densities in e.g. transition metals, especially if the results are based on LDA which may be not valid in the region of strongly varying densities.

\section{Acknowledgments}

The work is supported by the Committee for Scientific Research grant No. 204049101.

\section{References}

[1] H. Stachowiak, A. Rubaszek, in: Positrons at Metallic Surfaces, Ed. A. Ishii, in series Solid Stale Phenomena, Vol. 28-29, Trans. Tech. Publication, Aedermannsdorf 1993, p. 7.

[2] A. Rubaszek, J. Phys. Condens. Malter 1, 2141 (1989).

[3] S. Daniuk, M. Sob, A. Rubaszek, Phys. Rev. B 43, 2580 (1991).

[4] G. Kontrym-Sznajd, A. Rubaszek, submitted to AIP Conf. Proc. Series, Proc. 5lh Int. Workshop on Slow Positron Beam Techniques for Solids and Surfaces SLOPOS5; Phys. Rev. B 47 (1993), in press.

[5] E. Boroński, in: Positron Annihilation, Mater. Sci. Forum, Vol. 105-110, Eds. Zs. Kajcsos, Cs. Szeles, Trans. Tech. Publ., Aedermannsdorf 1992, p. 181.

[6] G. Kontrym-Sznajd, J. Majsnerowski, J. Phys., Condens. Matter 2, 9927 (1990).

[7] A. Rubaszek, H. Stachowiak, Phys. Status Solidi B 124, 159 (1984); Phys. Rev. B 38, 3846 (1988).

[8] E. Boroński, R.M. Nieminen, Phys. Rev. B 34, 3820 (1986). 